\title{
A SEMANTICALLY ENRICHED MULTIMODAL IMAGING APPROACH DEDICATED TO CONSERVATION AND RESTORATION STUDIES
}

\author{
Anthony Pamart ${ }^{1}$, Roxane Roussel ${ }^{2}$, Emile Hubert ${ }^{2}$, Alain Colombini ${ }^{2}$, Renato Saleri ${ }^{3}$, El Mustapha Mouaddib ${ }^{4}$, Yuly Castro $^{5}$, \\ Gaëtan Le Goïc 5 , Alamin Mansouri ${ }^{5}$ \\ ${ }^{1}$ Modèles et simulations pour l'Architecture et le Patrimoine, UMR 3495 CNRS/MC, MAP-GAMSAU, Marseille, France - \\ anthony.pamart@map.cnrs.fr \\ ${ }^{2}$ Centre Interdisciplinaire de Conservation et de Restauration du Patrimoine, LABCOM CICRP/MAP, Marseille, France - \\ roxane.roussel@cicrp.fr \\ ${ }^{3}$ Modèles et simulations pour l'Architecture et le Patrimoine, UMR 3495 CNRS/MC, MAP-ARIA, Lyon, France \\ ${ }^{4}$ MIS Laboratory, University of Picardie Jules Verne, Amiens, France \\ ${ }^{5}$ ImViA Laboratory, Université de Bourgogne Franche-Comté, Dijon, France
}

\section{Commission II}

KEY WORDS: multimodal-imaging, photogrammetry, lasergrammetry, multi-light image collection, RTI, technical photography, heritage sciences, collaborative web-service, semantic annotation, digital cartography, conservation, restoration

\begin{abstract}
:
For the past decades, Cultural Heritage $(\mathrm{CH})$ is commonly documented by digital-based imaging and analytical techniques. This documentation is used as a support by heritage scientists to study and help the preservation of $\mathrm{CH}$ objects. Multiple techniques or modalities are usually required and applied to complete the documentation and the possible diagnostic from it. In this paper we explored multimodal imaging strategies to survey, analyse and share semantically enriched digital replicas. Three challenging casestudies from the SUMUM research project aims to illustrate efficient multi-source approaches in multi-scalar, multi-temporal and multi-spectral contexts. From multimodal data acquisitions, a photogrammetric-based registration method (TACO) has been developed in order to exploit a 2D/3D semantic annotation process implemented into a $\mathrm{CH}$ oriented collaborative web platform (AIOLI). In the exemples showed, the structure and the content of the annotations work is based from condition reports provided by conservation and restoration experts. To this end, all the documentation gathered on $\mathrm{CH}$ objects are either directly merged by image based registration while complementary analysis can be spatially anchored to annotations as linked resources. The dissemination part is explored by built-in AIOLI's collaborative features or external Potree-based viewer, to enhance the accessibility of the final 3D annotated scenes for further expertises or wide-public events and purposes.
\end{abstract}

\section{INTRODUCTION}

One of the priorities in the field of Digital Cultural Heritage $(\mathrm{DCH})$ is to find and use strategically appropriate tools and methods to study and document the heritage objects. Conservation and restoration works imply the study of their appearance, material, dimension, physical or chemical behaviors and their interaction with the environment causing alteration through time. Multimodal imaging surveys are necessary, in order to provide effective support for diagnosis and condition reporting of those complex mechanisms. In our approach, Image based modeling (IBM) practices are envisioned to create thorough studies gathering in a common spatial reference system a rich documentation from complex and various multimodal imaging strategies. Considering the complexity and the uniqueness of $\mathrm{CH}$ objects, few scenarios were tested in which different heritage science experts intervened to build and share knowledge over a digital replica and its spatially oriented resources.

According to this research aim, this article presents and summarizes the work achieved in the SUMUM research project (founded by ANR-17-CE38-0004) aiming to explore cross examination of multimodal imaging for Conservation and Restoration purposes. One of the research axis focuses on building a collaborative semantic enrichment framework over spatially-oriented resources. The three case studies, each one corresponding to a multi-scale, multi-temporal or multi-spectral documentation approach are presented. The objective was to develop from ad-hoc multimodal acquisition campaigns, through dedicated processing toolbox and toward seamless integration into the AIOLI semantic annotation web platform
(Manuel et al. 2018) an integrated framework aligned with conservation and restoration works.

Our contribution is positioned to previous works cited in the section 2. The methodology developed is detailed in respective subsections in data acquisition in 3.1, registration in 3.2, annotation and visualisation 3.3 to 3.5 . Main outcome from each case study is outlined in subsections 4.1 to 4.3 and discussed in 4.4 before the concluding words in section 5 .

\section{RELATED WORKS}

For a decade, real-based modeling including several imaging techniques coming from passive or active sensing have been widely used in combinaison to survey, document and study $\mathrm{CH}$ sites and objects. Multi-source approaches (i.e. using different sensors, or techniques) are often required to complete the virtual reconstruction and improve cross-analysis (Barazzeti, 2010; Grifoni, 2018; Nocerino, 2018). However, since data fusion in this field is a challenging issue (Ramos, 2015; Adamopoulos, 2019), one lead is to explore co-registration and spatial annotation to improve the cross examination of multiple imaging techniques. Furthermore, Heritage Sciences and heritage management being multidisciplinary fields, current and innovative data to knowledge sharing and collaborative framework, are usually performed through web-based systems (3DHOP, Potree, ARCHES, AIOLI). Most recent works include solution like integrated framework (Gasparetto, 2021) or multilayered and/or relightable imaging (Jaspe 2021; Pamart 2019) exploration to enhance visual data interpretation of fused imaging modalities. 


\section{DEVELOPED METHODOLOGY}

\subsection{Multi modal acquisitions}

The experiment revolves around three case studies, from the modern period chosen for their challenging appearance, material and monumental dimensions causing troubles for one, several or each imaging techniques. Therefore, the difficulties imposed by those works of art required ad-hoc multi-modal acquisition strategies expressed through different documentation scenario : «Zett» by Victor Vasarely (Fig. 4) mainly focused on an multi-scale approach, "L'Arbre aux Serpents » by Niki de Saint-Phalle (Fig. 1) with multi-temporal data and «Expansion controlée » by César (Fig. 2) with a multispectral methodology.

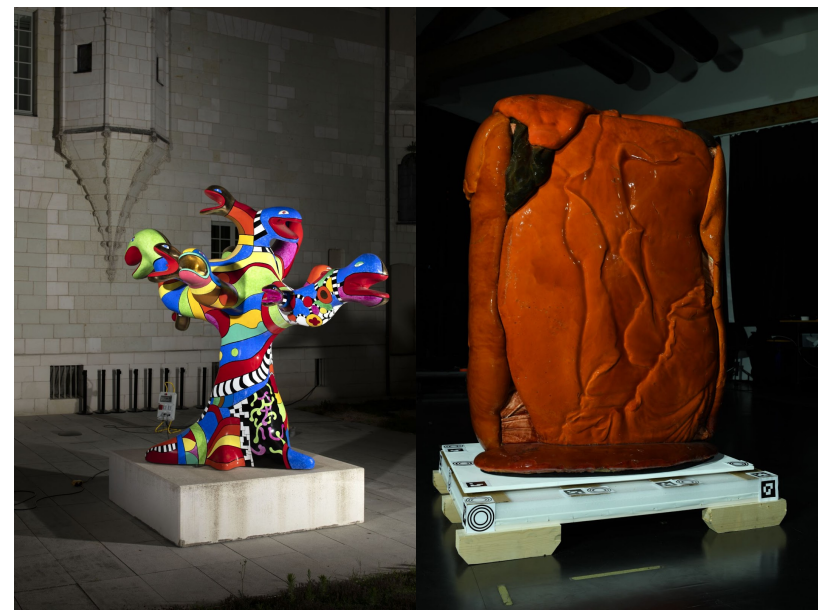

Figure 1 and 2. Photography of "L'Arbre aux serpents" by Niki de Saint-Phalle (left) and "Expansion contrôlée" by César (right)

Those case studies, exposed in indoor or outdoor environments were subjected to several acquisitions before, during and after restoration, to inspect and follow-up a wide range of alterations and decays documented and analyzed by heritage scientists. The 2D/3D imaging and analytical techniques performed are the most used in current practices (Bedford, 2017; Broadman, 2017; Dyer, 2018; Hanke, 2001) detailed hereafter and below in Figure. 3 :

- Terrestrial Laser Scanning (TLS) mostly used to extract "ground-truth" and GCP's

- Terrestrial Close-Range photogrammetry (T-CRP)

- Aerial (UAV-based) Close-Range photogrammetry (A-CRP)
- Multi Light Imaging Collection (MLIC) such as Multispectral RTI (MS-RTI) or Photometric Stereo (PS)

- Technical Photography (TP) in visible (VIS), raking light (RaK), infrared (IR), ultraviolet (UV) or cross-polarization (CP) setups.

- Documentary Photography (DOC), single or isolated pictures from archive or for documentation purposes

- Possible mixed or hybrid techniques like infrared or crosspolarized photogrammetry.

- In addition, analytical techniques such as Spectrocolorimeter (SC) and sampling analysis with FTIR (SP) were performed and integrated to deepen conservation and restoration knowledge (Fig. 5).

During the data acquisition campaigns, each scientific partner was carrying an expertise concerning an imaging or analysis technique, namely ; photogrammetry (MAP-GAMSAU and ARIA), lasergrammetry (MIS), MLIC (ImVia and MAPGAMSAU), Technical Photograph and other analytical techniques (CICRP). The global strategy was to anticipate since data capture the constraints to improve the cooperation between our respective techniques hence to increase the multimodal data fusion possibilities and performances.

\subsection{Photogrammetric based processing for spatial registration and geometry reconstruction}

The data processing was performed by Totally Automated Coregistration and Orientations (TACO) which is a MicMac based library automating incremental registration for multi-source $3 \mathrm{D}$ reconstruction. This Python-based package was developed within the SUMUM research project and previously presented (Pamart et Al., 2020). The code source of the beta release is available following this link; https://page.hn/5oot7b. In parallel, TACO is the photogrammetric engine to process image sets directly into AIOLI, however since the incremental mode hasn't been implemented yet in the web-service, the dataset were processed off-line then uploaded with a new import function.

The incremental processing is done as follows ; for a given initial image set (if possible a global and optimal acquisition) after-defined as master acquisition, a first scene reconstruction is generated (including cameras and geometry) on which other image sets (i.e other modalities) are incrementally co-registered among pre-oriented sets. The subset of scientific imaging presented for each case studies were automatically registered after several iterations in order to build, for the first time, a collaborative annotation framework from multimodal $2 \mathrm{D} / 3 \mathrm{D}$ scene.

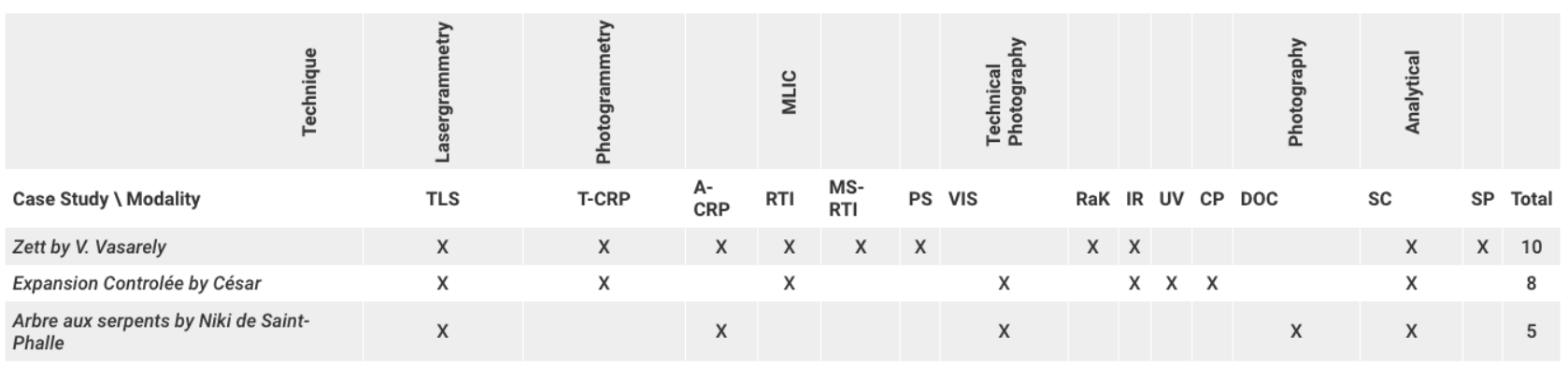

Figure 3. Synthetic overview of the multimodal data fusion performed on each case-study 


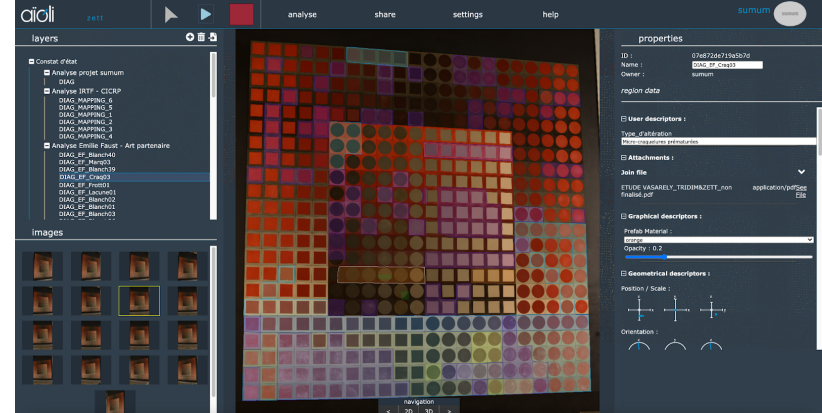

Figure 4. Digital cartography of the alterations within AIOLI on "Zett" data set

\subsection{Integration into AIOLI web-platform}

The AIOLI platform is reality-based $2 \mathrm{D} / 3 \mathrm{D}$ annotation system allowing to build semantically enriched digitization of heritage assets from a photogrammetric-friendly image set and spatial annotations coupled with additional resources. This web-service aims to create an innovative framework for the massive and large-scale collaborative $\mathrm{CH}$ documentation by linking features like image-based 3D reconstruction, 2D-3D spreading and correlation of semantic annotations, multi-layered analysis of qualitative and quantitative attributes.

Recently a new feature has been developed allowing to import any Micmac-based project to make it manageable with annotation process. In parallel, a conversion script has been developed using Metashape API to convert a project from this commercial solution into a compliant MicMac format therefore readable by AIOLI import function.

To this end, a compressed file containing a list of images and corresponding camera calibrations, the sparse and dense point clouds, GCP's 2D/3D correspondences and some essential structured metadata had to be assembled.

Then the ZIP files were uploaded onto the platform, as a preprocessed project to run a point cloud to images indexation method internal to AIOLI. Once this process is over, the semantic annotation could then begin on the oriented pictures, allowing in the meantime an automatic and seamless 2D/3D reprojection within the scene, as the background tasks.

\subsection{A collaborative annotation from the documentation provided by heritage scientists}

With the help of heritage scientists and following thoroughly the previous diagnosis, restoration reports and preliminary studies carried out, the key information were extracted and classified into a series of groups and layers replicated into the AIOLI projects (Fig. 4, 7, 8 and 9). A series of annotations were established, completed with linked documents and description sheets, in order to enrich the 2D/3D annotation (Fig. 5, 7 and 8). This step also included the collaborative framework : the project owner could allow users to intervene in the project, and create their own semantic annotations.

\subsection{Exploiting public 3D viewers for the dissemination of annotated projects}

Finally, an extended and/or public access had to be granted for every project. The aim is to allow experts and non-experts to explore the enriched documentation by navigating freely into an interactive $2 \mathrm{D} / 3 \mathrm{D}$ environment, as well as consulting the groups, layers and related annotations. Two visualization frameworks are explored, one tailored for research and $\mathrm{CH}$ experts purpose and one for wide public and museographic uses. The first solution is made possible natively in the AIOLI platform : if a project owner chooses to consider his project as a public project, a consultation link is provided. This link can be diffused and will only allow any heritage scientist to access and consult at any time the documentation gathered. This viewer allows any project owner to share with peers and observe a centralized and up-to-date project, forbidding any modification or removal. An example scene (Fig.4) is provided at the following URL; https://page.hn/qaxsdt. However, the experience to provide for non-experts is obviously different as the information shared has to be filtered to ease and guide their understanding. To this end, an external viewer is planned and will benefit from a more parametrizable Potree-based prototype (Fig. 5). This work-in-progress public viewer will allow users to compose from an Aioli's project a simplified scene. The aim is to create a sort of a story-telling subset made only of components that ease the interpretation or the understanding for a target audience.

Nonetheless, hyperlinked sources like above mentioned remains volatile especially when dealing with scientific data set available on a self-hosted research prototype. As the editable tiny-url provided above neither is long-term and stable resource nor compliant to FAIR principles, we are experimenting alternatives to enhance publicly available annotated 2D/3D data with perennial identifiers. A solution would be to attribute a Digital Object Identifier (DOI) to an Aioli scene itself or to rely on a more flexible system (e.g. ARK) to able to cite or refer to any sub-hierarchical elements (from group of layers to a single annotation) composing the scene.

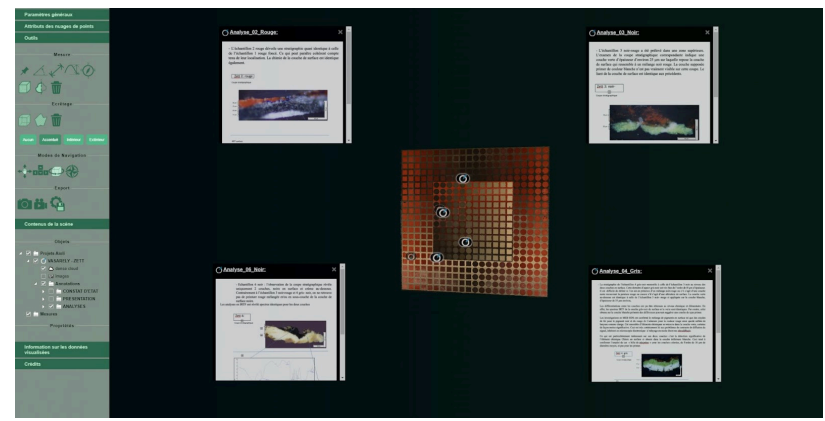

Figure 5. Complementary analysis linked to spatial annotation as joint-resources on Potree-based viewer exported from "Zett" Aioli project

\section{RESULTS AND DISCUSSION}

For the final stage of this research project, it has been decided to focus on different approaches emphasizing for each case study, a multimodal scenario. The data presented below do not reveal the real volume of data collected but only a subset selected for each case-study considering their relevance to the documentation scenario and issue revealed by the diagnosis of conservation state.

\subsection{The multi-scale approach illustrated by the "Zett » case-study from Victor Vasarely collection set}

Among the 36 monumental paintings exposed in the Vasarely foundation in Aix-en-Provence, 9 were selected and documented with multimodal imaging. Zett case-study was selected above all to lead this experimental worksite, because of its specific Nextel coating which raises interesting conservation issues (uncontrolled ageing) and having the benefit of increasing the texture of the surface more suitable for a photogrammetric-based annotation. Indeed, plain black and white, aluminium or glass based artworks were more difficult or impossible to acquire with all imaging techniques. To face the 


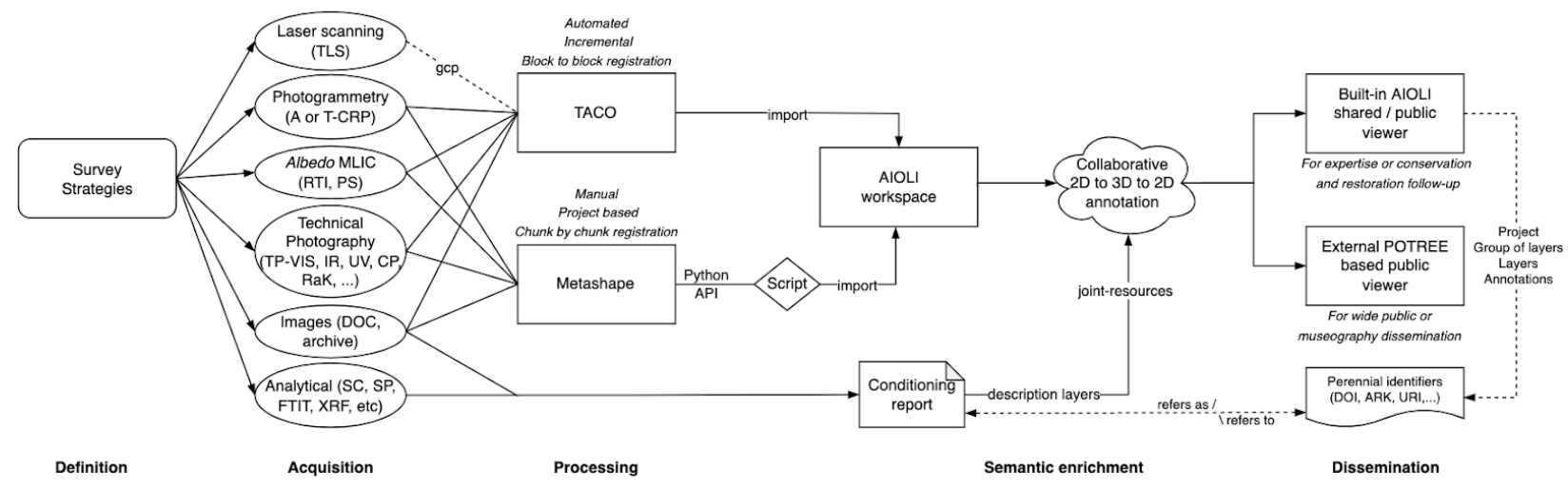

Figure 6. Overview of the semantically enriched workflow developed

dimension of this painting $(528 \times 528 \mathrm{~cm})$, the multi-scale approach was preferred to link the global conservation diagnosis completed with additional resources spatially linked through annotations. In the final project, 227 pictures were registered from 9 iterations :

- A T-CRP handled and convergent global acquisition

- A close-up A-CRP acquisition performed with indoor UAV flight

- A T-CRP macro acquisition performed with visible (VIS) + Infrared (IR) orthomosaic multispectral guiding system

- Semi-Raking light (S-RAK) pictures extracted from the Technical Photography (TP) documentation set

- Multi Light Imaging collection sets, including Multispectral RTI (MS-RTI), dome-based RTI and Photometric Stereo (PS)

The average residue of $1.24 \mathrm{px}$ on the global set is accurate enough considering the wide variability and the scalar deviation between the modal layers successfully co-registered (over $14 \mathrm{x}$ magnification of the GSD). The annotation structure includes one layer with the complete nomenclature of the painted cardboard elements. Another group of layers concerns their detachment level and alterations (whitening, lack, cracks etc). A last one is used to point the location of stratigraphic samples analyzed with microscopy and FTIR jointed to the annotations (Fig.5). This case-study is the most satisfying result obtained. Moreover the geometrical simplicity of this work of art enable to bypass several issues encountered and discussed in the next exemples.

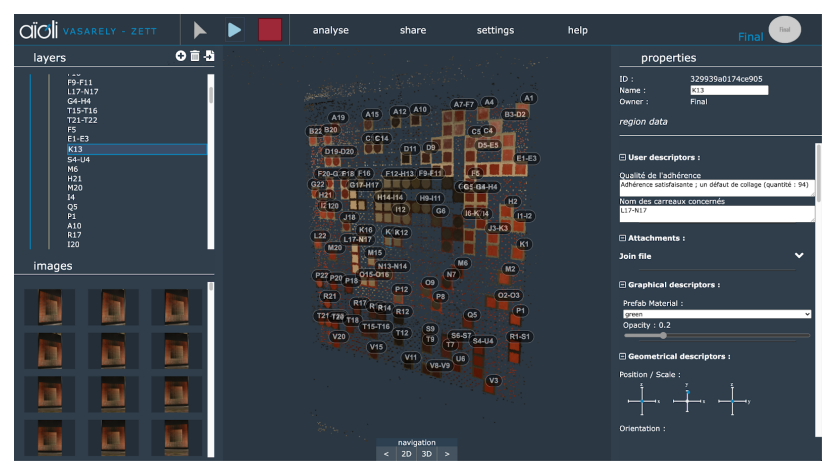

Figure 7. Revealing detachments through 3D dense pointcloud annotations on « Zett»

\subsection{The multi-temporal approach illustrated by the «Arbre aux serpents " case-study from Niki de Saint-Phalle collection set}

This case-study is dedicated to a multi-temporal approach because the chance was given to make a photogrammetric survey just before and right after a complete restoration of this severely damaged monumental sculpture exposed outdoors. As the surface was pickled, rewhiten and repainted by the original craftsman, we seized the opportunity to capture and combine altered and restored states to create an optimal follow-up documentation set. The final dataset is composed of 178 pictures registered in 5 iterations defining 4 modal layers :

- An A-CRP set extracted from a UAV mission in 2016 before restoration

- An A-CRP set extracted from a UAV mission in 2018 after restoration

- Pictures of the visible (VIS) Technical Photography-based (TP) documentation set

- Few isolated documentary photographs (DOC) from 2012 but not taken for photogrammetric purposes

The average residue of $0.97 \mathrm{px}$ is decent considering the important changing of aspect between the different temporal states. It has been decided to rely on the first photogrammetric survey to define the master acquisition because the alteration of the surface actually improved the quality of registration and reconstruction. Despite this choice, the complex snake shape and the appearance was a struggling challenge for $3 \mathrm{D}$ dense matching reconstruction, resulting in a noisy and incomplete point-set. To cope with this issue, we combined the image-based aerial point-set with the terrestrial laser-based one - also suboptimal because of reflective coating and metallic painting) acquired after restoration to serve as 3D basis for Aioli's annotation process. One layer concerns a condition report from photogrammetry and the documentary survey acquisition before the restoration. A second layer aims to locate spectrocolorimetric analysis (with CIELAB coordinates joint as linked resources) performed after restoration, as shown in Fig.8. Some mismatches occurred and remain with the registration of the non-photogrammetric images from 2012. In addition, some annotations are inconsistent in depth because of holes in the point cloud which is a known and yet unresolved issue of Aioli's indexation and propagation internal process on complex 3D shapes. 


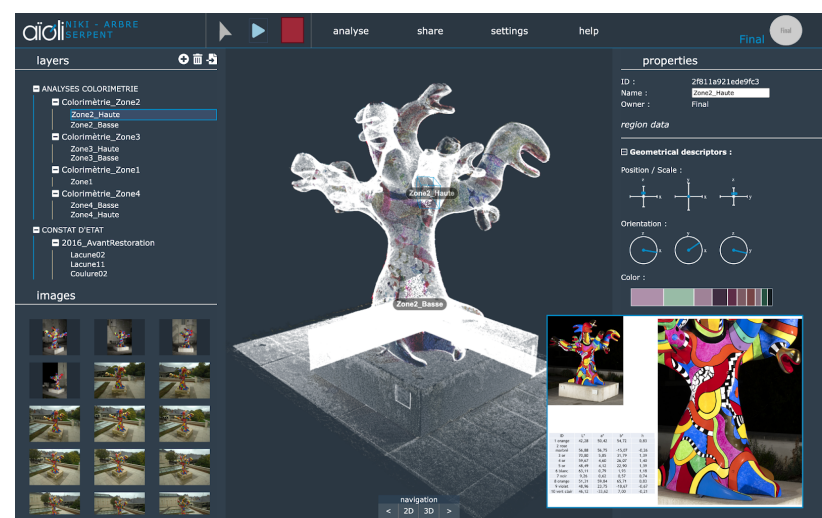

Figure 8. Correlation of joint spectroscopic analysis and color-palette from the dense cloud on «Arbre aux serpents »

\subsection{The multi-spectral approach illustrated by the " Expansion contrôlée" case-study from Cesar collection set}

This case study approached by multi-spectral scenario was by far the most challenging and complicated object to document leading to more limited outcomes. This sculpture made of composite material (polyurethane foam over polystyrene core structure) even with moderate dimensions $(153 \times 116 \times 80 \mathrm{~cm})$ complexified acquisition strategies because of its blobby shape compound with pronounced glossiness (variable thickness of polyester varnish). Fortunately, we had the opportunity to have at disposal the sculpture in a wide and empty photographic studio so as to optimize the capture set-up and benefit of fully controlled lighting. The final dataset is completed by 116 pictures registered in 9 iterations defined by the following modalities :

\section{- A T-CRP global capture with cross-polarisation (CP) set-up}

- Several albedo viewpoints (14) extracted from automated RTI dome capture set

- An extended Technical Photography set composed of visible (VIS), infrared reflected (IR) and ultraviolet fluorescence (UV) capture (indirectly registered from VIS camera positions).

As expected we get a higher average residue of $1.66 \mathrm{px}$ probably caused by the wide radiometric deviation of these combined modalities. Indeed, it was the most complicated object to survey and treat, its appearance required a cross-polarized master photogrammetric set to cope with the specular variation issue. In spite of all, the registration of RTI capture and multispectral photography to document such complex appearance was challenging but necessary to highlight major elements of the condition report (past restorations and some alterations remain only visible in multispectral domain as shown in Fig. 9). The annotation is composed of a layer recalling intervention areas from a restoration made in 2017 completed with a group of layers pointing visual and mechanical alterations, still visible in the actual state. The laser point-set (enhance with reflectance intensities) was also used as reference because of poor performance of $3 \mathrm{D}$ dense matching from $\mathrm{CP}$ sources.

\subsection{Limits and discussions}

All over the operative chain (Fig. 6), from acquisition to public dissemination numerous issues have been encountered, a critical review is proposed hereafter. The first difficulty is related to the

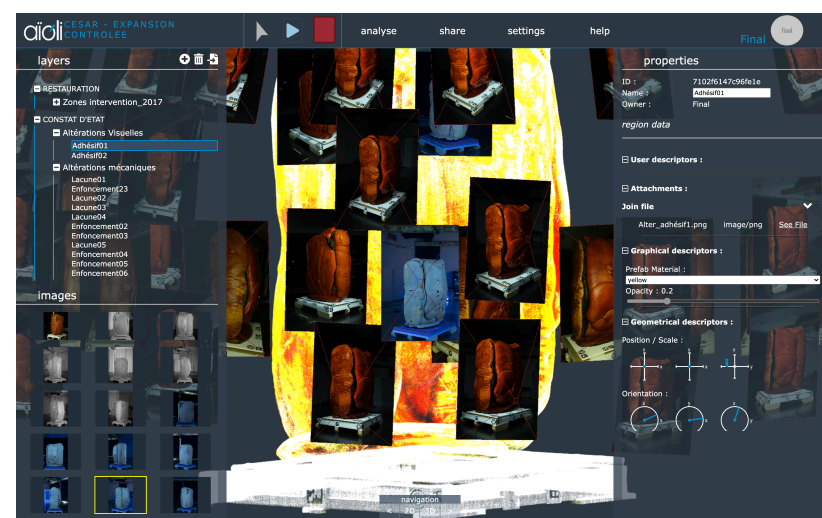

Figure 9. Registration of UV fluorescence to reveal and propagate past-restoration annotations on « Expansion Contrôlée 》

necessity to define and anticipate ad-hoc multimodal data acquisition protocols to help and prevent data registration and data fusion issues. The main outcome is to identify and agree on the best strategy that embraces multiple imaging expertises, requirements and constraints to optimize each modality. One can notice that many other techniques like multispectral or hyperspectral imaging or other technologies of laser scanning commonly used for cultural heritage application were not tested during this study. Other difficulties emerged during data processing as our photogrammetric based registration (TACO) is robust to modalities variation to a certain point. Unfortunately, complex environments, materials or shapes common in $\mathrm{CH}$ field like in our case-studies still remain challenging objects to document even with a strategic multimodal imaging survey. In our case, the solution proposed showed a good balance performance between automation, versatility, velocity and robustness while guaranteeing accuracy in both pose estimations and dense reconstruction considering the sparse and variable modalities in input. Finally, dealing with complex multimodal scenarios like the ones presented is increasing the difficulties in terms of data provenance, management, file format and interoperability etc. Those issues are emphasized when the aim is to integrate those data into a user-friendly and domain oriented framework, like AIOLI. As expected, multiplying the sources, also imply the multiplication of the technical aspects (change and variation of resolution, orientation, density, accuracy) as many ways of creating i) new issues and bugs on operative part but also ii) updated methodological challenges for an effective data fusion (degree of confidence of input and measured performance of the merged output). Concerning this point, this experiment revealed the need and the challenge associated toward seamless data streams, accessible at scientific community level, from large-scale cloud infrastructure. According to the work achieved some developments still have to be planned to improve efficiency and user experience of processing, annotation and viewer components. However thanks to this work, we are one step forward to perform seamless multi-source annotation within AIOLI on genuinely complex multimodal contexts.

\section{CONCLUSIONS}

In this article, we presented a complete methodology to construct, share and potentially re-use semantically enriched digital replica build of over multi-source acquisition strategies based on commonly used imaging techniques for $\mathrm{CH}$ applications. By the challenging case studies chosen and the results obtained we demonstrated the versatility and robustness of TACO in the context of data registration and fusion from 
genuine and complex multimodal imaging surveys. Once the photogrammetric reconstruction is achieved by TACO or converted with Metashape-based script, the data structured into an AIOLI compliant format can be imported into this web platform to support collaborative and shareable 2D/3D semantic annotation scenarii. Most of the imaging techniques performed on-site were successfully handled by processing and annotation components, if not, they were included as joint ressource spatially linked to the annotation. A special care has been taken to conform all the information from the diagnosis and condition report established from the conservation and restoration experts. Most of the annotation layouts presented reproduce the conventional cartographic work made either on Technical Photography or Orthophotography basis while the hierarchical description (group of layers, layers, user defined descriptors) is derived from the structured content of conservation reports. To this end, the resulting 3D model can act as a portal to access the spatially oriented resources, and all related analysis and documentation collected overtime during conservation and restoration works. On one side, the dissemination of such semantically enriched digital heritage assets can be achieved directly with the Aioli Viewer if the aim is to share and open the data to heritage scientists. On the other side, the external viewer will allow to disseminate and expose to the wide public and for museographic purposes the progress and the innovation made nowadays by the Digital Heritage scientific community.

\section{ACKNOWLEDGEMENTS}

This work was funded by the ANR SUMUM project, grant reference ANR-17-CE38-0004 of the French Agence Nationale de la Recherche. The authors would like to acknowledge the stakeholders of the case-studies illustrating this paper, namely ; the "Zett» painting of Victor Vasarely from the Fondation Vasarely in Aix-en-Provence, the sculpture "L'arbre aux serpents » from Niki de Saint Phalle from the Musée des Beaux-arts d'Angers and the "Expansion controlée" " sculpture from César in the courtesy of the Musée d'Art Contemporain de Marseille. Equally, the authors would like to thank :

- J. Toupance for her help during his internship for the acquisition campaign

- F. Morlet for his devoted work to develop TACO

- T. Zanetti for his external viewer experiment in progress and the illustration kindly provided

- The authors of the condition reports for their agreement to transpose and disseminate their diagnosis via Aioli

\section{REFERENCES}

Adamopoulos, E., \& Rinaudo, F. 2019. 3D interpretation and fusion of multidisciplinary data for heritage science: A review. In 27th CIPA International Symposium-Documenting the Past for a Better Future (Vol. 42, No. 2, pp. 17-24). International Society for Photogrammetry and Remote Sensing.

Aïoli - A reality-based 3D annotation cloud platform for the collaborative documentation of cultural heritage artefacts. n.d. http://www.aioli.cloud/

Barazzetti, L., Remondino, F., Scaioni, M., Lo Brutto, M., Rizzi, A., \& Brumana, R. 2010. Geometric and radiometric analysis of paintings. International Archives of Photogrammetry, Remote Sensing and Spatial Information Sciences, 38(Part 5).
Bedford, J. 2017. Photogrammetric Applications for Cultural Heritage: Guidance for Good Practice. Historic England.

Boardman, C., \& Bryan, P. 2018. 3D laser scanning for heritage: Advice and guidance on the use of laser scanning in archaeology and architecture. Historic England.

Dyer, J., Verri, G., \& Cupitt, J. 2013. Multispectral Imaging in Reflectance and Photo-induced Luminscence Modes: A User Manual. British Museum.

Gasparetto, F., \& Baratin, L. 2021. Conservation 4.0. Possible Guidlines for Standardising the Documental Process for Artistic Heritage. The International Archives of Photogrammetry, Remote Sensing and Spatial Information Sciences, 46, 257-264.

Grifoni, E., Legnaioli, S., Nieri, P., Campanella, B., Lorenzetti, G., Pagnotta, S., Poggialini F. \& Palleschi, V. 2018. Construction and comparison of 3D multi-source multi-band models for cultural heritage applications. Journal of Cultural Heritage, 34, 261-267.

Hanke, L. D. 2001. Handbook of analytical methods for materials. Materials Evaluation and Engineering Inc., Plymouth, 35-38.

Jaspe-Villanueva, A., Ahsan, M., Pintus, R., Giachetti, A., Marton, F., \& Gobbetti, E. 2021. Web-based Exploration of Annotated Multi-Layered Relightable Image Models. Journal on Computing and Cultural Heritage (JOCCH), 14(2), 1-29.

Manuel, A., and Al. 2018. A semi-automatic 2D/3D annotation framework for the geometric analysis of heritage artefacts. In 2018 3rd Digital Heritage International Congress (DigitalHERITAGE) held jointly with 2018 24th International Conference on Virtual Systems \& Multimedia (VSMM 2018) (pp. 1-7). IEEE.

Nocerino, E., Rieke-Zapp, D. H., Trinkl, E., Rosenbauer, R., Farella, E., Morabito, D., \& Remondino, F. 2018. Mapping VIS and UVL imagery on 3D geometry for non-invasive, noncontact analysis of a vase. International Archives of the Photogrammetry, Remote Sensing and Spatial Information Sciences, 42, 773-780.

Pamart, A., Guillon, O., Vallet, J.-M. and De Luca, L., 2016. Toward a multimodal photogrammetric acquisition and processing methodology for monitoring conservation and restoration studies. In: Proceedings of the 14th Eurographics Workshop on Graphics and Cultural Heritage, Eurographics Association, pp. 207-210.

Pamart, A., and Al. 2019. A complete framework operating spatially oriented RTI in a $3 \mathrm{D} / 2 \mathrm{D}$ cultural heritage documentation and analysis tool. ISPRS - International Archives of the Photogrammetry, Remote Sensing and Spatial Information Sciences XLII-2/W9.

Pamart, A., Morlet, F., De Luca, L., \& Veron, P. 2020. A Robust and Versatile Pipeline for Automatic Photogrammetric-Based Registration of Multimodal Cultural Heritage Documentation. Remote Sensing, 12(12), 2051.

Ramos, M. M., \& Remondino, F. 2015. Data fusion in cultural heritage-A review. The International Archives of Photogrammetry, Remote Sensing and Spatial Information Sciences, 40(5), 359. 